\title{
Analysis of a SIRI Epidemic Model with Distributed Delay and Relapse
}

\author{
Abdelhai Elazzouzi $^{1, *}$, Abdesslem Lamrani Alaoui ${ }^{2}$, Mouhcine Tilioua ${ }^{2}$, Delfim F. M. Torres ${ }^{3}$ \\ ${ }^{1}$ Department of MPI, University Sidi Mouhamed Ben Abdellah, FP Taza, LSI Laboratory, Morocco \\ ${ }^{2}$ Department of Mathematics, Moulay Ismail University of Meknes, FST Errachidia, M2I Laboratory, MAMCS Group, Morocco \\ ${ }^{3}$ Center for Research and Development in Mathematics and Applications (CIDMA), Department of Mathematics, University of Aveiro, \\ 3810-193 Aveiro, Portugal
}

\begin{abstract}
We investigate the global behaviour of a SIRI epidemic model with distributed delay and relapse. From the theory of functional differential equations with delay, we prove that the solution of the system is unique, bounded, and positive, for all time. The basic reproduction number $R_{0}$ for the model is computed. By means of the direct Lyapunov method and LaSalle invariance principle, we prove that the disease free equilibrium is globally asymptotically stable when $R_{0}<1$. Moreover, we show that there is a unique endemic equilibrium, which is globally asymptotically stable, when $R_{0}>1$.
\end{abstract}

Keywords Global stability, Nonlinear incidence function, Distributed delay, Lyapunov functionals, Relapse.

\section{AMS 2010 subject classifications 34D23, 92D30}

DOI: $10.19139 /$ soic-2310-5070-831

\section{Introduction}

In recent years, great attention has been paid to the study of SIR type models, which have been formulated to describe the propagation and evolution of some human or animal diseases. In such models, the population is subdivided into compartments or classes, in particular the compartment of susceptible $(S)$, the compartment of infective $(I)$, and the compartment of recovered individuals $(R)[18,19]$. When recovered individuals may experience a relapse of the disease, due to an incomplete treatment or due to the reactivation of a latent infection, and then re-enter the class of infective, a SIRI model is more convenient to model the dynamic of the diseases. Herpes, which can be transmitted by close physical or sexual contact, tuberculosis, and malaria, are three epidemics that have been modeled by SIRI systems [14, 17, 22, 25, 26]. Often, the transmission of the infection in the population is modelled by an incidence function, which has taken many forms in the literature [3, 5, 9]. Most epidemiological models focus on an incidence function without delay [15]. They assume that infection occur instantaneously once there is a contact between an infectious individual and a susceptible one. On the other hand, some models incorporate an incidence function with discrete or distributed delays to model latency [4, 10, 13].

In the analysis of local or global asymptotic stability properties, it is common to use Lyapunov's second method, also called the direct method of Lyapunov. It is a robust tool that allows to determine the stability of a system without explicitly integrating the differential equation. For details, see, e.g., [1, 8, 11, 12, 20]. Several works include relapse. In [21], a constant population SIRI model with the incidence function $S(t) I(t)$ is analysed. In [16], an extension of the model in [21] for herpes viral infections is investigated. It is proved that both disease-free and endemic equilibria of the model are globally asymptotically stable [16]. In [5], a SIRI epidemic model with the incidence rate of infection $C(S) f(I)$ is studied. Sufficient conditions for the local stability of the equilibria

\footnotetext{
${ }^{*}$ Correspondence to: A. Elazzouzi (Email: abdelhai.elazzouzi@usmba.ac.ma), Sidi Mouhamed Ben Abdellah, FP Taza, LSI Laboratory,
} Morocco.

ISSN 2310-5070 (online) ISSN 2311-004X (print)

Copyright (C) 2019 International Academic Press 


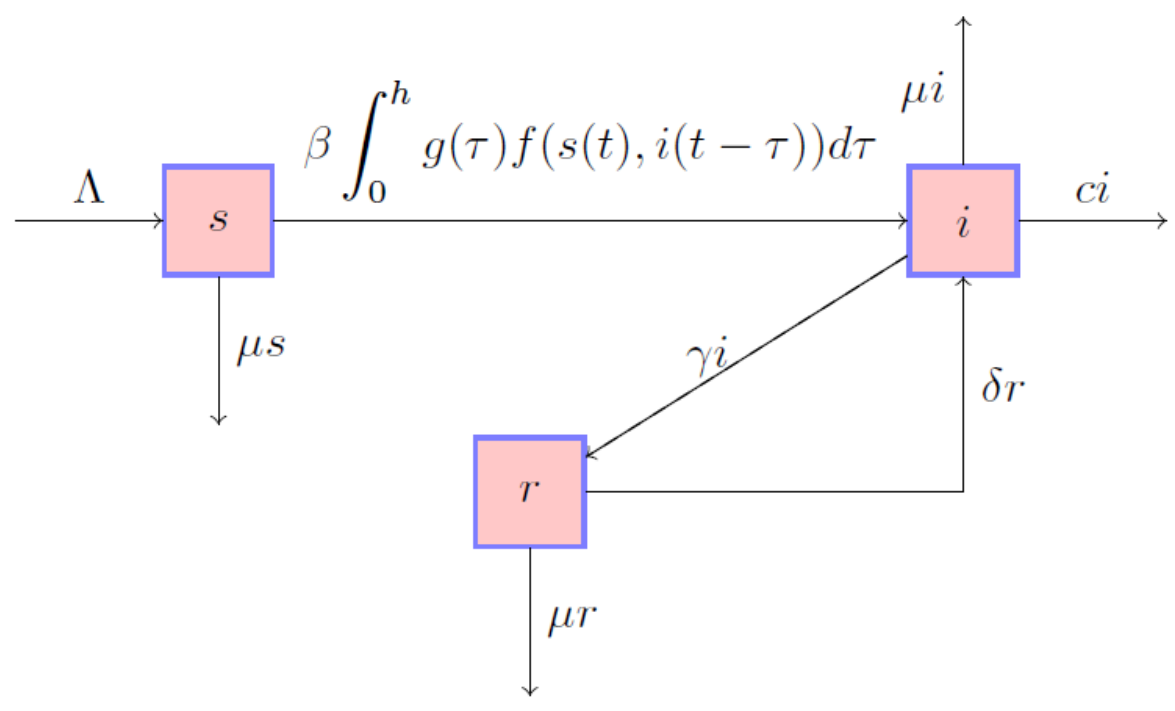

Figure 1. Flow diagram of the disease transmission

are given by using Lyapunov's second method and, under suitable monotonicity conditions, global stability is obtained. In [24], the global stability of a SIRI model with constant recruitment, disease-induced death, and bilinear incidence rate, is discussed. Here, we consider a SIRI model with relapse, a distributed delay, and a general nonlinear incidence function. The direct method of Lyapunov is used to prove global asymptotic stability for any steady state.

The paper is organized as follows. The mathematical model under consideration is formulated in Section 2. In Section 3, we establish its well-posedness. More precisely, we prove positivity and boundedness of the solution (Theorem 1). The basic reproduction number and the disease-free equilibrium $E_{0}$ are also determined (Theorem 2). In Section 4, we provide a mathematical analysis of the model. In particular, the global stability of the disease-free equilibrium and the global stability of the endemic equilibrium are shown (Theorems 3 and 4). Two numerical examples with an incidence function satisfying the assumptions considered in the previous sections are given in Section 5. We finish the paper with Section 6 of concluding remarks and some perspectives for future research.

\section{The mathematical model}

We consider a general SIRI epidemic model with distributed delay and relapse. The flow diagram of the disease transmission is given in Figure 1, which corresponds to the dynamics described by the system of equations (1):

$$
\left\{\begin{array}{l}
\frac{d s(t)}{d t}=\Lambda-\mu s(t)-\beta \int_{0}^{h} g(\tau) f(s(t), i(t-\tau)) d \tau, \\
\frac{d i(t)}{d t}=\beta \int_{0}^{h} g(\tau) f(s(t), i(t-\tau)) d \tau-(\mu+c+\gamma) i(t)+\delta r(t), \\
\frac{d r(t)}{d t}=\gamma i(t)-(\mu+\delta) r(t),
\end{array}\right.
$$

where $s(t), i(t)$, and $r(t)$ denote, respectively, the number of susceptible, infective, and recovered individuals at time $t$. The parameters of model (1) are summarized in Table 1. Individuals leave the susceptible class at a rate

$$
\int_{0}^{h} g(\tau) f(s(t), i(t-\tau)) d \tau
$$


Table 1. Description of the parameters of the SIRI model (1).

\begin{tabular}{c|l}
\hline Parameter & Biological meaning \\
\hline$\Lambda$ & the population recruitment rate \\
$\mu$ & the population natural death rate \\
$\gamma$ & the natural recovery rate of infective individuals \\
$c$ & the population death rate caused by infection \\
$\beta$ & the transmission coefficient \\
$\delta$ & the relapse rate \\
\hline
\end{tabular}

where $h$ represents the maximum time taken to become infectious and $g$ denotes the fraction of vector population in which the time taken to become infectious is $\tau$ (that is, the incubation period distribution), which is assumed to be a non-negative continuous function on $[0, h]$. Moreover, and without loss of generality, we assume that

$$
\int_{0}^{h} g(\tau) d \tau=1
$$

Otherwise, we consider

$$
\underline{g}=\frac{g}{\int_{0}^{h} g(\tau) d \tau} \quad \text { and } \quad \underline{\beta}=\beta \int_{0}^{h} g(\tau) d \tau
$$

instead of $g$ and $\beta$, respectively. The initial conditions for system (1) are given for $\theta \in[-h, 0]$ by

$$
s(\theta)=\Phi_{1}(\theta), \quad i(\theta)=\Phi_{2}(\theta), \quad r(\theta)=\Phi_{3}(\theta)
$$

with $\Phi=\left(\Phi_{1}, \Phi_{2}, \Phi_{3}\right) \in C^{+} \times C^{+} \times C^{+}$, where $C^{+}=C\left([-h, 0], \mathbb{R}^{+}\right)$is the non-negative cone of $C$. Here, $C:=C([-h, 0], \mathbb{R})$ denotes the space of continuous functions mapping $[-h, 0]$ into $\mathbb{R}$, equipped with the supnorm. Let $s(0)=s_{0}>0, i(0)=i_{0}>0$, and $r(0)=r_{0}>0$. Our main objective is to investigate the global stability of the SIRI model (1). For that, we construct suitable Lyapunov functionals.

\section{The well-posedness of the model and its basic reproduction number}

Let $f: \mathbb{R}_{+}^{2} \rightarrow \mathbb{R}_{+}$be a continuously differentiable function in the interior of $\mathbb{R}_{+}^{2}$, satisfying the following hypotheses:

$\left(H_{1}\right) f(s, i)$ is a strictly monotone increasing function of $s \geqslant 0$ for any fixed $i>0$ and a monotone increasing function of $i>0$ for any fixed $s \geqslant 0$;

$\left(H_{2}\right) \phi(s, i)=\frac{f(s, i)}{i}$ is a bounded and monotone decreasing function of $i>0$ for any fixed $s \geqslant 0$;

$\left(H_{3}\right) f(0, i)=f(s, 0)=0$ for all $s, i \geqslant 0$.

Then, the right side of (1) is locally Lipschizian and we get, from the classical theory of ODEs with delay, local existence and uniqueness of solution for (1), i.e., existence and uniqueness for all $t \in[0, \delta]$ for some $\delta \geq 0[6,7]$. It is also easy to see that system (1) has always a disease-free equilibrium

$$
E_{0}=\left(\frac{\lambda}{\mu}, 0,0\right) .
$$

We begin by proving that our model (1) is not only mathematically but also biologically well-posed: all feasible solutions of system (1) are bounded and positive. 


\section{Theorem 1}

Let $(s(t), i(t), r(t))$ be any solution of model (1) with positive initial condition $\left(s_{0}, i_{0}, r_{0}\right)$. Then,

1. every solution of (1) starting from $\left(s_{0}, i_{0}, r_{0}\right)$ remains positive for all $t \geq 0$,

2. the set

$$
\Omega=\left\{(s, i, r) \in \mathbb{R}^{3}: s>0, i>0, r>0, s+i+r \leq \frac{\Lambda}{\mu}\right\}
$$

is positively invariant with respect to system (1).

Proof

Assume, by contradiction, that the first item of our result is false. Let $t_{1}=\min \{t: s(t) i(t)=0\}$. Assume that $s\left(t_{1}\right)=0$, which implies that $i(t) \geq 0$ for all $0 \leq t \leq t_{1}$. Let

$$
A=\min _{0 \leq t \leq t_{1}}\left\{\frac{\Lambda}{s(t)}-\mu-\beta \int_{0}^{h} g(\tau) \frac{f(s(t), i(t-\tau))}{s(t)} d \tau\right\} .
$$

It follows that $\frac{d}{d t} s(t) \geq A s(t)$. Therefore, $s\left(t_{1}\right) \geq s(0) \exp \left(A t_{1}\right)>0$. This contradicts $s\left(t_{1}\right)=0$. With a similar argument, we see that $i\left(t_{1}\right)=0$ is a contradiction. This proves that $s(t)>0$ and $i(t)>0$ for all $t \geq 0$. On the other hand, from the third equation of (1), one has $\frac{d}{d t} r(t) \geq-(\mu+\delta) r(t)$, which implies

$$
r(t) \geq r(0) \exp (-(\mu+\delta) t)>0 .
$$

We have just proved that any solution $(s(t), i(t), r(t))$ is positive. Now, let $N(t)=s(t)+i(t)+r(t)$. Then,

$$
\frac{d}{d t} N(t)=\Lambda-\mu N(t)-c i(t)
$$

It follows that

$$
\frac{d}{d t} N(t) \leq \Lambda-\mu N(t)
$$

Now, let $V$ be the unique solution of the initial value problem

$$
\left\{\begin{array}{l}
\frac{d}{d t} V(t)=\Lambda-\mu V(t) \text { for } t>0, \\
V(0)=N(0) .
\end{array}\right.
$$

Then, $V$ is given by

$$
V(t)=\frac{\Lambda}{\mu}(1-\exp (-\mu t))+N(0) \exp (-\mu t)
$$

and, by the comparison theorem (see Theorem 5 in Appendix), it follows that

$$
N(t) \leq \frac{\Lambda}{\mu}(1-\exp (-\mu t))+N(0) \exp (-\mu t) .
$$

This implies that the solution is bounded and, by the blow-up phenomena, the solution exists and is defined for all $t \geq 0$. Moreover, for $t$ going to $+\infty$, we have

$$
0 \leq N(t) \leq \frac{\Lambda}{\mu}
$$

Since the solution is positive and bounded, we conclude that $\Omega$ is positively invariant with respect to (1). 
Theorem 2

The basic reproduction number of model (1) is given by

$$
R_{0}=\frac{\beta(\mu+\delta) \partial_{2} f\left(E_{0}\right)}{(\mu+\delta)(\mu+c+\gamma)-\delta \gamma}
$$

where $\partial_{2} f\left(E_{0}\right)$ denotes the partial derivative of $f$ with respect to its second argument $i$ at the disease-free equilibrium $E_{0}$ given by (2).

Proof

We obtain the basic reproduction number by means of the next generation method as given in [23]. Let $x=(i, r, s)$. Then, it follows from system (1) that

$$
\frac{d x}{d t}=\mathcal{F}-\nu
$$

where

$$
\mathcal{F}=\left(\begin{array}{c}
\beta \int_{0}^{h} g(\tau) f(s(t), i(t-\tau)) d \tau \\
0 \\
0
\end{array}\right)
$$

and

$$
\nu=\left(\begin{array}{c}
(\mu+c+\gamma) i(t)-\delta r(t) \\
-\gamma i(t)+(\mu+\delta) r(t) \\
\beta \int_{0}^{h} g(\tau) f(s(t), i(t-\tau)) d \tau-\Lambda+\mu s(t)
\end{array}\right) .
$$

At the disease-free equilibrium $E_{0}$,

$$
D \mathcal{F}\left(E_{0}\right)=\left(\begin{array}{cc}
F & O_{2,1} \\
O_{1,2} & 0
\end{array}\right), \quad D \nu\left(E_{0}\right)=\left(\begin{array}{cc}
V & O_{2,1} \\
J_{1} & J_{2}
\end{array}\right),
$$

where the infection matrix $F$ and the transition matrix $V$ are given by

$$
F=\left(\begin{array}{cc}
\beta \partial_{2} f\left(E_{0}\right) & 0 \\
0 & 0
\end{array}\right) \quad \text { and } \quad V=\left(\begin{array}{cc}
\mu+c+\gamma & -\delta \\
-\gamma & \mu+\delta
\end{array}\right)
$$

The inverse of $V$ is

$$
V^{-1}=\frac{1}{(\mu+\delta)(\mu+c+\gamma)-\delta \gamma}\left(\begin{array}{cc}
\mu+\delta & \delta \\
\gamma & \mu+c+\delta
\end{array}\right) .
$$

Thus, the next generation matrix for system (1) is

$$
F V^{-1}=\frac{\beta \partial_{2} f\left(E_{0}\right)}{(\mu+\delta)(\mu+c+\gamma)-\delta \gamma}\left(\begin{array}{cc}
\mu+\delta & \delta \\
0 & 0
\end{array}\right) .
$$

The basic reproduction number $R_{0}$ is the spectral radius of the matrix $F V^{-1}$, and the result follows. 


\section{Analysis of the model}

In this section, we prove that there exists a unique endemic equilibrium when the basic reproduction number given by Theorem 2 is greater than one (Lemma 1), and we obtain conditions for which the disease-free equilibrium and the endemic equilibrium are globally asymptotically stable (Theorems 3 and 4, respectively).

\subsection{Existence of an endemic equilibrium}

In this section, we establish existence and uniqueness of an endemic equilibrium.

Lemma 1

If $R_{0}>1$, then system (1) admits a unique endemic equilibrium $E^{*}=\left(s^{*}, i^{*}, r^{*}\right)$.

Proof

We look for the solutions $\left(s^{*}, i^{*}, r^{*}\right)$ of equations $\frac{d s}{d t}=0, \frac{d i}{d t}=0$, and $\frac{d r}{d t}=0$. First note that $\frac{d s}{d t}+\frac{d i}{d t}=0$ implies

$$
\Lambda-\mu s^{*}-(\mu+c+\gamma) i^{*}+\frac{\delta \gamma}{\mu+\delta} i^{*}=0
$$

and so

$$
s^{*}=\frac{\Lambda}{\mu}-\frac{(\mu+c+\gamma)-\frac{\delta \gamma}{\mu+\delta}}{\mu} i^{*} .
$$

Let $H$ be the function defined from $\mathbb{R}^{+}$to $\mathbb{R}$ by

$$
H(i)=\beta \frac{f\left(\frac{\Lambda}{\mu}-\frac{(\mu+c+\gamma)-\frac{\delta \gamma}{\mu+\delta}}{\mu} i, i\right)}{i}-(\mu+c+\gamma)+\frac{\delta \gamma}{\mu+\delta} .
$$

It follows that $H$ satisfies

$$
\lim _{i \rightarrow 0^{+}} H(i)=\beta \partial_{2} f\left(E_{0}\right)-(\mu+c+\gamma)+\frac{\delta \gamma}{\mu+\delta}=\left((\mu+c+\gamma)-\frac{\delta \gamma}{\mu+\delta}\right)\left(R_{0}-1\right)>0
$$

and

$$
H\left(\frac{\Lambda(\mu+\delta)}{(\mu+\delta)(\mu+c+\gamma)-\delta \gamma}\right)=\frac{\delta \gamma}{\mu+\delta}-(\mu+c+\gamma)<0
$$

Then, by the intermediate value theorem, there exists at least a $i^{*}$ such that

$$
0<i^{*}<\frac{\Lambda(\mu+\delta)}{(\mu+\delta)(\mu+c+\gamma)-\delta \gamma} \quad \text { and } \quad H\left(i^{*}\right)=0 .
$$

Moreover, hypothesis $\left(H_{2}\right)$ implies that $H$ is a strictly monotone decreasing function on $\mathbb{R}^{+}$. Then, we conclude with the existence and uniqueness of $i^{*}$ such that

$$
0<i^{*}<\frac{\Lambda(\mu+\delta)}{(\mu+\delta)(\mu+c+\gamma)-\delta \gamma} \quad \text { and } \quad H\left(i^{*}\right)=0 .
$$

Furthermore, since $\int_{0}^{h} g(\tau) d \tau=1$, it follows that

$$
\beta \int_{0}^{h} g(\tau) f\left(s^{*}, i^{*}\right) d \tau=\Lambda-\mu s^{*}
$$


and

$$
\beta \int_{0}^{h} g(\tau) f\left(s^{*}, i^{*}\right) d \tau=(\mu+c+\gamma) i^{*}-\delta r^{*}
$$

where

$$
r^{*}=\frac{\gamma}{\mu+\delta} i^{*}
$$

Therefore, $E^{*}=\left(s^{*}, i^{*}, r^{*}\right)$ is the unique endemic equilibrium of system (1).

\subsection{Global stability of the disease-free equilibrium}

We define a Lyapunov functional, showing the global asymptotic stability of the disease-free equilibrium $E_{0}$ of system (1).

\section{Theorem 3}

Assume that the hypotheses $\left(H_{1}\right)$ and $\left(H_{2}\right)$ hold. Then, the disease free equilibrium $E_{0}$ of system (1) is globally asymptotically stable if, and only if, $R_{0} \leq 1$.

Proof

Let

$$
w(t)=s(t)-s_{0}-\int_{\frac{\Lambda}{\mu}}^{s(t)} \lim _{i \rightarrow 0^{+}} \frac{f\left(s_{0}, i(t)\right)}{f(\sigma, i(t))} d \sigma+i(t)+k \int_{0}^{h} g(t) \int_{0}^{\tau} i(t-u) d u d \tau+\frac{\delta}{\mu+\delta} r(t),
$$

where

$$
k=\frac{(\mu+\delta)(\mu+c+\gamma)-\delta \gamma}{\mu+\delta}
$$

We have

$$
\begin{aligned}
\frac{d w(t)}{d t}= & \left(1-\lim _{i \rightarrow 0^{+}} \frac{f\left(s_{0}, i(t)\right)}{f(s(t), i(t))}\right)\left(\Lambda-\mu s(t)-\beta \int_{0}^{h} g(\tau) f(s(t), i(t-\tau)) d \tau\right) \\
& +\beta \int_{0}^{h} g(\tau) f(s(t), i(t-\tau)) d \tau-(\mu+c+\gamma) i(t)+\delta r(t) \\
& +\frac{\delta}{\mu+\delta}(\gamma i(t)-(\mu+\delta) r(t))+k \int_{0}^{h} g(t)(i(t)-i(t-\tau)) d \tau .
\end{aligned}
$$

It follows that

$$
\begin{aligned}
\frac{d w(t)}{d t}= & \mu\left(1-\lim _{i \rightarrow 0^{+}} \frac{f\left(s_{0}, i(t)\right)}{f(s(t), i(t))}\right)\left(s_{0}-s(t)\right) \\
& +\beta \lim _{i \rightarrow 0^{+}} \frac{f\left(s_{0}, i(t)\right)}{f(s(t), i(t))} \int_{0}^{h} g(\tau) f(s(t), i(t-\tau) d \tau-(\mu+c+\gamma) i(t) \\
& +\delta r(t)+\frac{\delta}{\mu+\delta}(\gamma i(t)-(\mu+\delta) r(t))+k \int_{0}^{h} g(t)(i(t)-i(t-\tau)) d \tau \\
= & \mu\left(1-\lim _{i \rightarrow 0^{+}} \frac{f\left(s_{0}, i(t)\right)}{f(s(t), i(t))}\right)\left(s_{0}-s(t)\right) \\
& +\int_{0}^{h} g(\tau)\left(\beta \frac{f(s(t), i(t-\tau))}{k i(t-\tau)} \lim _{i \rightarrow 0^{+}} \frac{f\left(s_{0}, i(t)\right)}{f(s(t), i(t))}-1\right) k i(t-\tau) d \tau
\end{aligned}
$$

Therefore,

$$
\beta \frac{f(s(t), i(t-\tau))}{k i(t-\tau)} \lim _{i \rightarrow 0^{+}} \frac{f\left(s_{0}, i(t)\right)}{f(s(t), i(t))} \leq \frac{\beta}{k} \frac{\partial f\left(s_{0}, i(t)\right)}{\partial i}=R_{0} .
$$


Then,

$$
\frac{d w(t)}{d t}=\mu\left(1-\lim _{i \rightarrow 0^{+}} \frac{f\left(s_{0}, i(t)\right)}{f(s(t), i(t))}\right)\left(s_{0}-s(t)\right)+\int_{0}^{h} g(\tau)\left(R_{0}-1\right) k i(t-\tau) d \tau .
$$

We conclude that $\frac{d w(t)}{d t} \leq 0$. Hence, $w$ is a Lyapunov functional for the system (1). Namely, $w^{\prime} \leq 0$ for all $(s, i, r) \in \Omega$, where $\Omega$ denotes the interior of $\Omega$. Thus, $w^{\prime}=0$ if and only if $(s, i, r)=\left(s_{0}, 0,0\right)$. This shows that the largest invariant subset where $w^{\prime}=0$ is the singleton $\left\{E_{0}\right\}$. By La Salle's invariance principle, $E_{0}$ is globally asymptotically stable. This completes the proof.

\subsection{Global stability of the endemic equilibrium}

Now we look for the global asymptotic stability of the endemic equilibrium $E^{*}$ of system (1). To this end, we construct a suitable Lyapunov functional. We have the following result.

Theorem 4

Assume that the hypotheses $\left(H_{1}\right)$ and $\left(H_{2}\right)$ hold. If $R_{0}>1$, then the unique endemic equilibrium $E^{*}$ given by Lemma 1 is globally asymptotically stable.

Proof

Let $G(x)=x-1-\ln (x)$. We have $G(x) \geq 0$ if $x>0$ and $G(x)=0$ if $x=1$. Let us consider the following Lyapunov functional: $V(t)=V_{1}(t)+V_{2}(t)+V_{3}(t)$, where

$$
\begin{gathered}
V_{1}(t)=s(t)-s^{*}-\int_{s^{*}}^{s(t)} \frac{f\left(s^{*}, i^{*}\right)}{f\left(\sigma, i^{*}\right)} d \sigma+i(t)-i^{*}-i^{*} \ln \left(\frac{i(t)}{i^{*}}\right), \\
V_{2}(t)=\beta f\left(s^{*}, i^{*}\right) \int_{0}^{h} g(\tau) \int_{0}^{\tau} G\left(\frac{i(t-u)}{i^{*}}\right) d u d \tau, \quad V_{3}(t)=\frac{\delta}{\mu+\delta}\left(r(t)-r^{*}-r^{*} \ln \left(\frac{r(t)}{r^{*}}\right)\right) .
\end{gathered}
$$

Then,

$$
\begin{aligned}
& \frac{d V_{1}(t)}{d t}=\left(1-\frac{f\left(s^{*}, i^{*}\right)}{f\left(s(t), i^{*}\right)}\right)(\Lambda \\
& \left.-\mu s(t)-\beta \int_{0}^{h} g(\tau) f(s(t), i(t-\tau)) d \tau\right) \\
& \quad+\left(1-\frac{i^{*}}{i(t)}\right)\left(\beta \int_{0}^{h} g(\tau) f(s(t), i(t-\tau)) d \tau-(\mu+c+\gamma) i(t)+\delta r(t)\right) .
\end{aligned}
$$

We also have

$$
\frac{d V_{2}(t)}{d t}=\beta f\left(s^{*}, i^{*}\right) \int_{0}^{h} g(\tau)\left(G\left(\frac{i(t)}{i^{*}}\right)-G\left(\frac{i(t-\tau)}{i^{*}}\right)\right) d \tau
$$

and

$$
\frac{d V_{3}(t)}{d t}=\frac{\delta}{\mu+\delta}\left(1-\frac{r^{*}}{r(t)}\right)(\gamma i(t)-(\mu+\delta) r(t))
$$

One can see that

$$
G\left(\frac{i(t)}{i^{*}}\right)-G\left(\frac{i(t-\tau)}{i^{*}}\right)=\frac{i(t)}{i^{*}}-\frac{i(t-\tau)}{i^{*}}+\ln \left(\frac{i(t-\tau)}{i(t)}\right) .
$$


Thus,

$$
\left\{\begin{array}{l}
\Lambda=\mu s^{*}+\beta f\left(s^{*}, i^{*}\right), \\
\beta f\left(s^{*}, i^{*}\right)=(\mu+c+\gamma) i^{*}-\frac{\delta \gamma}{\mu+\delta} i^{*} \\
\gamma i^{*}=(\mu+\delta) r^{*}
\end{array}\right.
$$

It follows that

$$
\begin{aligned}
\frac{d V(t)}{d t}= & \left(1-\frac{f\left(s^{*}, i^{*}\right)}{f\left(s(t), i^{*}\right)}\right)\left(\mu s^{*}+\beta f\left(s^{*}, i^{*}\right)-\mu s(t)\right) \\
& -\beta\left(1-\frac{f\left(s^{*}, i^{*}\right)}{f\left(s(t), i^{*}\right)}\right) \int_{0}^{h} g(\tau) f(s(t), i(t-\tau)) d \tau \\
& +\left(1-\frac{i^{*}}{i(t)}\right)\left(\beta \int_{0}^{h} g(\tau) f(s(t), i(t-\tau)) d \tau-(\mu+c+\gamma) i(t)\right) \\
& +\left(1-\frac{i^{*}}{i(t)}\right) \delta r(t) \\
& +\beta f\left(s^{*}, i^{*}\right) \int_{0}^{h} g(\tau)\left(\frac{i}{i^{*}}-\frac{i(t-\tau)}{i^{*}}+\ln \left(\frac{i(t-\tau)}{i(t)}\right)\right) d \tau \\
& +\frac{\delta}{\mu+\delta}\left(1-\frac{r^{*}}{r(t)}\right)(\gamma i(t)-(\mu+\delta) r(t)) \\
= & \mu\left(1-\frac{f\left(s^{*}, i^{*}\right)}{f\left(s(t), i^{*}\right)}\right)\left(s^{*}-s(t)\right) \\
& +\beta f\left(s^{*}, i^{*}\right) \int_{0}^{h} g(\tau)\left(\frac{f(s(t), i(t-\tau))}{f\left(s(t), i^{*}\right)}-\frac{i^{*}}{i(t)} \frac{f(s(t), i(t-\tau))}{f\left(s^{*}, i^{*}\right)}\right) d \tau \\
& +\beta f\left(s^{*}, i^{*}\right) \int_{0}^{h} g(\tau)\left(2-\frac{f\left(s^{*}, i^{*}\right)}{f\left(s(t), i^{*}\right)}-\frac{i(t)}{i^{*}}\right) d \tau \\
& +\beta f\left(s^{*}, i^{*}\right) \int_{0}^{h} g(\tau)\left(\frac{i}{i^{*}}-\frac{i(t-\tau)}{i^{*}}+\ln \left(\frac{i(t-\tau)}{i(t)}\right)\right) d \tau \\
& +\left(2 \frac{\delta \gamma}{\mu+\delta} i^{*}-\delta \frac{i^{*}}{i(t)} r(t)-\frac{\delta \gamma}{\mu+\delta} \frac{i(t)}{r(t)} r^{*}\right) \\
& +(t)
\end{aligned}
$$

\section{Using}

$$
\ln \left(\frac{i(t-\tau)}{i(t)}\right)=\ln \left(\frac{f\left(s^{*}, i^{*}\right)}{f\left(s(t), i^{*}\right)}\right)+\ln \left(\frac{i^{*}}{i(t)} \frac{f(s(t), i(t-\tau))}{f\left(s^{*}, i^{*}\right)}\right)+\ln \left(\frac{i(t-\tau)}{i^{*}} \frac{f\left(s(t), i^{*}\right)}{f(s(t), i(t-\tau))}\right)
$$


then

$$
\begin{aligned}
& \frac{d V(t)}{d t}=\mu\left(1-\frac{f\left(s^{*}, i^{*}\right)}{f\left(s(t), i^{*}\right)}\right)\left(s^{*}-s(t)\right) \\
& +\beta f\left(s^{*}, i^{*}\right) \int_{0}^{h} g(\tau)\left(1-\frac{f\left(s^{*}, i^{*}\right)}{f\left(s(t), i^{*}\right)}+\ln \left(\frac{f\left(s^{*}, i^{*}\right)}{f\left(s(t), i^{*}\right)}\right)\right) d \tau \\
& +\beta f\left(s^{*}, i^{*}\right) \int_{0}^{h} g(\tau)\left(1-\frac{i^{*}}{i(t)} \frac{f(s(t), i(t-\tau))}{f\left(s^{*}, i^{*}\right)}\right) d \tau \\
& +\beta f\left(s^{*}, i^{*}\right) \int_{0}^{h} g(\tau) \ln \left(\frac{i^{*}}{i(t)} \frac{f(s(t), i(t-\tau))}{f\left(s^{*}, i^{*}\right)}\right) d \tau \\
& +\beta f\left(s^{*}, i^{*}\right) \int_{0}^{h} g(\tau)\left(1-\frac{i(t-\tau)}{i^{*}} \frac{f\left(s(t), i^{*}\right)}{f(s(t), i(t-\tau))}\right) d \tau \\
& +\beta f\left(s^{*}, i^{*}\right) \int_{0}^{h} g(\tau) \ln \left(\frac{i(t-\tau)}{i^{*}} \frac{f\left(s(t), i^{*}\right)}{f(s(t), i(t-\tau))}\right) d \tau \\
& +\beta f\left(s^{*}, i^{*}\right) \int_{0}^{h} g(\tau)\left(\frac{i(t-\tau)}{i^{*}} \frac{f\left(s(t), i^{*}\right)}{f(s(t), i(t-\tau))}-1\right) d \tau \\
& +\beta f\left(s^{*}, i^{*}\right) \int_{0}^{h} g(\tau)\left(-\frac{i(t-\tau)}{i^{*}}+\frac{f(s(t), i(t-\tau))}{f\left(s(t), i^{*}\right)}\right) d \tau \\
& -\frac{\delta \gamma}{\mu+\delta} \frac{i(t)}{r(t)} r^{*}\left(\frac{i^{*} r(t)}{i(t) r^{*}}-1\right)^{2}
\end{aligned}
$$

The hypotheses $\left(H_{1}\right)$ and $\left(H_{2}\right)$ ensure that

$$
\frac{i(t-\tau)}{i^{*}} \frac{f\left(s(t), i^{*}\right)}{f(s(t), i(t-\tau))}-1-\frac{i(t-\tau)}{i^{*}}+\frac{f(s(t), i(t-\tau))}{f\left(s(t), i^{*}\right)} \leq 0
$$

From the hypothesis $\left(H_{1}\right)$, we have

$$
\mu\left(1-\frac{f\left(s^{*}, i^{*}\right)}{f\left(s(t), i^{*}\right)}\right)\left(s^{*}-s(t)\right) \leq 0
$$

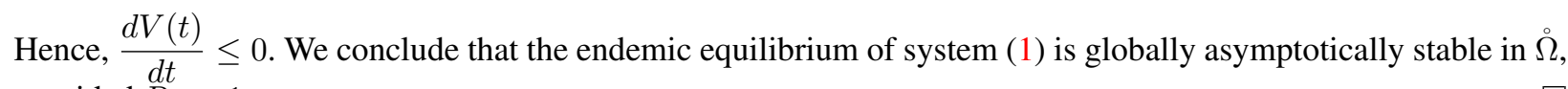
provided $R_{0}>1$. 


\section{Numerical simulations}

In this section, we carry out some numerical simulations to illustrate the obtained theoretical results. Consider the following delayed SIRI epidemic model with distributed time delay and relapse:

$$
\left\{\begin{array}{l}
\frac{d s(t)}{d t}=\Lambda-\mu s(t)-\beta \int_{0}^{h} \frac{e^{-\tau}}{1-e^{-h}} s(t) i(t-\tau) d \tau \\
\frac{d i(t)}{d t}=\beta \int_{0}^{h} \frac{e^{-\tau}}{1-e^{-h}} s(t) i(t-\tau) d \tau-(\mu+c+\gamma) i(t)+\delta r(t) \\
\frac{d r(t)}{d t}=\gamma i(t)-(\mu+\delta) r(t)
\end{array}\right.
$$

with initial conditions

$$
\Phi_{1}(\theta)=\sin (0.5 \theta)+150, \quad \Phi_{2}(\theta)=\sin (10 \theta)+20, \quad \Phi_{3}(\theta)=0, \quad-h \leq \theta \leq 0,
$$

or

$$
\Phi_{1}(\theta)=\cos (5 \theta)+200, \quad \Phi_{2}(\theta)=10 \sin (\theta)+30, \quad \Phi_{3}(\theta)=70, \quad-h \leq \theta \leq 0 .
$$

From Figure 2, we see that if $R_{0} \leq 1$, then, biologically speaking, the disease dies out from the population: the solution $(s(t), i(t), r(t))$ of (3) converges to the disease free equilibrium $E_{0}=\left(\frac{\Lambda}{\mu}, 0,0\right)$. Hence, $E_{0}$ is globally asymptotically stable.

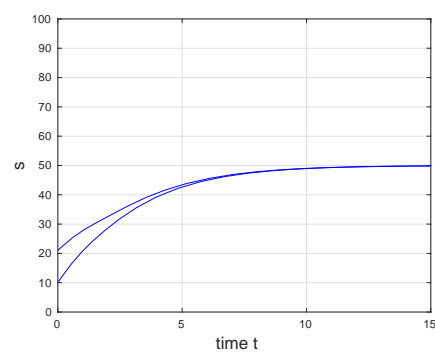

(a) $s(t)$

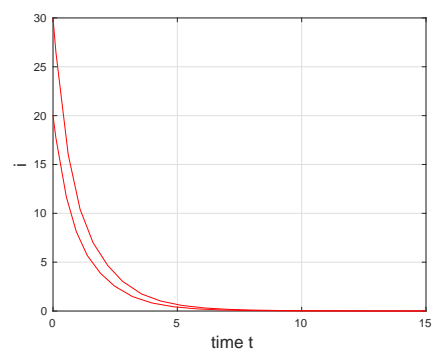

(b) $i(t)$

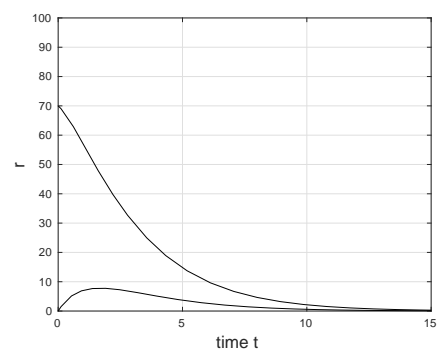

(c) $r(t)$

Figure 2. Trajectories of system (3) with time delay $h=2$, initial conditions (4) and (5), and parameters $\Lambda=20, \mu=0.4$, $\beta=0.02, c=0.1, \delta=0.006$, and $\gamma=0.7$, for which $R_{0}=0.8406<1$, converging to the disease free equilibrium $E_{0}$.

Figure 3 illustrates the case when $R_{0}>1$. In such situation, the solution $(s(t), i(t), r(t))$ of (3) converges to the endemic equilibrium $E^{*}$. Thus, the unique endemic equilibrium is globally asymptotically stable, which means, biologically, that the disease persists but is controlled.

\section{Concluding remarks}

We investigated a SIRI epidemic model (1) with distributed delay and relapse. The basic reproduction number $R_{0}$ was computed, which determines the existence of an equilibrium for the model. Precisely, when $R_{0} \leq 1$, then model (1) has one unique disease-free equilibrium $E_{0}$, while for $R_{0}>1$ it has a disease free equilibrium $E_{0}$ and a unique endemic equilibrium $E^{*}$. We proved, with the help of the direct Lyapunov method, that all steady states of (1) are globally asymptotically stable: the disease free equilibrium is globally asymptotically stable for $R_{0} \leq 1$; when $R_{0}>1$, then we established that there is a unique endemic equilibrium which is globally asymptotically stable. As future work, we plan to study a related model with distributed relapse. This is under current investigation and will be addressed elsewhere. 


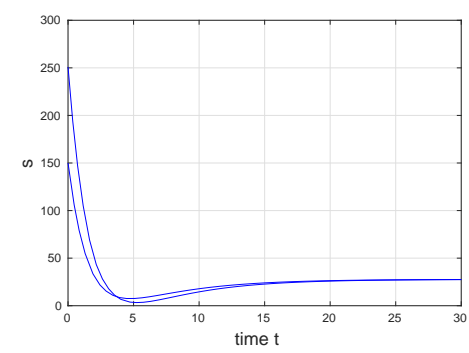

(a) $s(t)$

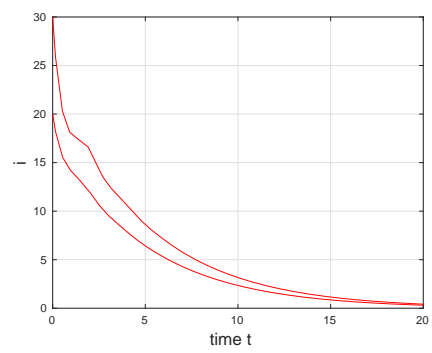

(b) $i(t)$

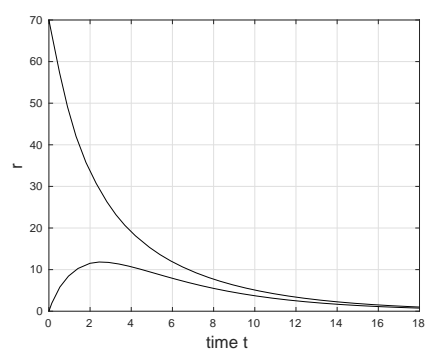

(c) $r(t)$

Figure 3. Trajectories of system (3) with time delay $h=2$, initial conditions (4) and (5), and parameters $\Lambda=18, \mu=0.65$, $\beta=0.2, c=0.77, \delta=0.02$, and $\gamma=0.75$, for which $R_{0}=2.2923>1$, converging to the endemic equilibrium $E^{*}$.

\section{Appendix}

\section{Theorem 5 (See [2])}

Let $f, g: A \rightarrow \mathbb{R}$, with $A \subset \mathbb{R}^{2}$ an open set, be continuous Lipschitz functions with respect to the second argument such that $f(t, x) \leq g(t, x)$ for all $(t, x) \in A$. Moreover, let $\left(t_{0}, y_{0}\right)$ and $\left(t_{0}, v_{0}\right)$ be two admissible initial states with $y_{0} \leq v_{0}$. Then, if $y, v: I \rightarrow \mathbb{R}$ are, respectively, the solutions of the Cauchy problems

$$
\left\{\begin{array}{l}
\frac{d y}{d t}=f(t, y(t)), \\
y\left(t_{0}\right)=y_{0}
\end{array}\right.
$$

and

$$
\left\{\begin{array}{l}
\frac{d v}{d t}=g(t, v(t)), \\
v\left(t_{0}\right)=v_{0}
\end{array}\right.
$$

where $I$ is the common interval of existence, then $y(t) \leq v(t)$ for all $t \in I$ and $t \geq t_{0}$.

\section{Acknowledgements}

This work was partially supported by FCT through the R\&D unit CIDMA, reference UID/MAT/04106/2019, and by project PTDC/EEI-AUT/2933/2014 (TOCCATA). The authors would like to thank two Reviewers for their critical remarks and precious suggestions, which helped them to improve the quality and clarity of the manuscript.

\section{REFERENCES}

1. E. Beretta, T. Hara, W. Ma and Y. Takeuchi, Global asymptotic stability of an SIR epidemic model with distributed time delay, Nonlinear Anal. 47 (2001), no. 6, 4107-4115.

2. M. Budinčević, A comparison theorem of differential equations, Novi Sad J. Math. 40 (2010), no. 1, 55-56.

3. Y. Enatsu, E. Messina, Y. Nakata, Y. Muroya, E. Russo and A. Vecchio, Global dynamics of a delayed SIRS epidemic model with a wide class of nonlinear incidence rates, J. Appl. Math. Comput. 39 (2012), no. 1-2, 15-34.

4. Y. Enatsu, Y. Nakata and Y. Muroya, Global stability of SIRS epidemic models with a class of nonlinear incidence rates and distributed delays, Acta Math. Sci. Ser. B (Engl. Ed.) 32 (2012), no. 3, 851-865.

5. P. Georgescu and $\mathrm{H}$. Zhang, A Lyapunov functional for a SIRI model with nonlinear incidence of infection and relapse, Appl. Math. Comput. 219 (2013), no. 16, 8496-8507.

6. J. K. Hale, Ordinary differential equations, Wiley-Interscience, New York, 1969.

7. J. Hale, Theory of functional differential equations, second edition, Springer-Verlag, New York, 1977. 
8. A. Korobeinikov, Lyapunov functions and global stability for SIR and SIRS epidemiological models with non-linear transmission, Bull. Math. Biol. 68 (2006), no. 3, 615-626.

9. A. Korobeinikov and P. K. Maini, A Lyapunov function and global properties for SIR and SEIR epidemiological models with nonlinear incidence, Math. Biosci. Eng. 1 (2004), no. 1, 57-60.

10. Y. Kuang, Delay differential equations with applications in population dynamics, Mathematics in Science and Engineering, 191, Academic Press, Inc., Boston, MA, 1993.

11. J. P. LaSalle, The stability of dynamical systems, Society for Industrial and Applied Mathematics, Philadelphia, PA, 1976.

12. J. LaSalle and S. Lefschetz, Stability by Liapunov's direct method, with applications, Mathematics in Science and Engineering, Vol. 4, Academic Press, New York, 1961.

13. C.-H. Li, C.-C. Tsai and S.-Y. Yang, Analysis of the permanence of an SIR epidemic model with logistic process and distributed time delay, Commun. Nonlinear Sci. Numer. Simul. 17 (2012), no. 9, 3696-3707.

14. Q. Liu, D. Jiang, T. Hayat and B. Ahmad, Stationary distribution and extinction of a stochastic SIRI epidemic model with relapse, Stoch. Anal. Appl. 36 (2018), no. 1, 138-151.

15. J. P. Mateus, P. Rebelo, S. Rosa, C. M. Silva and D. F. M. Torres, Optimal control of non-autonomous SEIRS models with vaccination and treatment, Discrete Contin. Dyn. Syst. Ser. S 11 (2018), no. 6, 1179-1199. arXiv:1706.06843

16. H. N. Moreira and Y. Wang, Global stability in an $S \rightarrow I \rightarrow R \rightarrow I$ model, SIAM Rev. 39 (1997), no. 3, 496-502.

17. B. G. S. A. Pradeep, W. Ma and W. Wang, Stability and Hopf bifurcation analysis of an SEIR model with nonlinear incidence rate and relapse, J. Stat. Manag. Syst. 20 (2017), no. 3, 483-497.

18. A. Rachah and D. F. M. Torres, Analysis, simulation and optimal control of a SEIR model for Ebola virus with demographic effects, Commun. Fac. Sci. Univ. Ank. Sér. A1 Math. Stat. 67 (2018), no. 1, 179-197. arXiv: 1705.01079

19. M. Sekiguchi, E. Ishiwata and Y. Nakata, Dynamics of an ultra-discrete SIR epidemic model with time delay, Math. Biosci. Eng. 15 (2018), no. 3, 653-666.

20. Y. Takeuchi, W. Ma and E. Beretta, Global asymptotic properties of a delay $S I R$ epidemic model with finite incubation times, Nonlinear Anal. 42 (2000), no. 6, Ser. A: Theory Methods, 931-947.

21. D. Tudor, A deterministic model for herpes infections in human and animal populations, SIAM Rev. 32 (1990), no. 1, 136-139.

22. P. van den Driessche, L. Wang and X. Zou, Modeling diseases with latency and relapse, Math. Biosci. Eng. 4 (2007), no. 2, $205-219$.

23. P. van den Driessche and J. Watmough, Reproduction numbers and sub-threshold endemic equilibria for compartmental models of disease transmission, Math. Biosci. 180 (2002), 29-48.

24. C. Vargas-De-León, On the global stability of infectious diseases models with relapse, Abstraction \& Application 9 (2013), 50-61.

25. R. Xu, Global dynamics of a delayed epidemic model with latency and relapse, Nonlinear Anal. Model. Control 18 (2013), no. 2, $250-263$.

26. H. Zhang, J. Xia and P. Georgescu, Multigroup deterministic and stochastic SEIRI epidemic models with nonlinear incidence rates and distributed delays: a stability analysis, Math. Methods Appl. Sci. 40 (2017), no. 18, 6254-6275. 